Joana Alves ${ }^{1}$

Ana Dutra ${ }^{1}$

Ângela Maia ${ }^{1}$
${ }^{1}$ Escola de Psicologia, Universidade do Minho. Campus de Gualtar. 4710-057 Braga Portugal. angelam@psi.uminho.pt

\section{História de adversidade, saúde e psicopatologia em reclusos: comparação entre homens e mulheres}

\author{
History of adversity, health and psychopathology \\ among prisoners: comparison between men and women
}

\begin{abstract}
Adversity in childhood, risk behaviors and psychopathology are highly prevalent phenomena in inmate populations and have a strong impact on health. Knowing the differences in these variables between the sexes is most important in order to develop appropriate intervention strategies in a prison context. By administering the Socio-demographic and Life History Questionnaire and the Brief Symptoms Inventory, we sought to characterize adverse childhood experiences and relate them to risk behaviors and to psychopathological symptoms, and study the differences between the 65 male and 42 female detainees in Portuguese prison establishments. Men and women report a complex web of adversity in childhood. In a range of ten possible categories, a medium value of $5.05(D P=2.63)$ in total adversity for women and $2.63(D P=2.18)$ for men was encountered, with the prevalence being significantly higher within the female population $(Z=$ -4.33; $p=.000)$. A high prevalence of risk behaviors and psychopathological symptoms was found in both groups, the latter being higher among females. We concluded that the differences between men and women calls for in depth studies in order to provide guidelines for intervention projects in specific populations.
\end{abstract}

Key words Prison, Health, Psychopathology, Risk behaviors, Adversity
Resumo Adversidade na infância, comportamentos de risco e psicopatologia são fenómenos muito prevalentes na população reclusa e com forte impacto na saúde. Conhecer as diferenças entre sexos, no que diz respeito a tais variáveis, é de elevada importância no sentido de adequar estratégias de intervenção em contexto prisional. Utilizando o Questionário Sociodemográfico e História de Vida, o Questionário de Adversidade na Infância e o Brief Symptons Inventory, procuramos caracterizar a adversidade na infância, os comportamentos de risco e as dimensões psicopatológicas, e averiguar as diferenças entre 65 homens e 42 mulheres reclusos em estabelecimentos prisionais Portugueses. Homens e mulheres relatam um quadro complexo de adversidade na infância. Num total possível de dez categorias, verificamos uma média de adversidade total de 5.05 $(D P=2.63)$ para as mulheres e de 2.63 (DP = 2.18) para os homens, sendo a prevalência significativamente mais elevada junto da população feminina $(Z=-4.33$; $p=.000)$. Foi ainda encontrada uma elevada prevalência de comportamentos de risco e de sintomatologia psicopatológica em ambos os grupos, sendo esta última superior nas mulheres. Concluímos que as diferenças entre sexos devem ser estudadas para guiarem a adequação dos projetos de intervenção junto de populações específicas.

Palavras-chave Prisão, Saúde, Psicopatologia, Comportamentos de risco, Adversidade 


\section{Introdução}

Nos últimos anos o estudo da relação entre experiências adversas na infância e sintomas físicos e psicológicos na idade adulta tem ganho especial importância, não só pelo impacto que essas experiências têm na saúde física e mental ao longo do ciclo de vida, mas também pelo facto de os indivíduos com história mais adversa terem maior probabilidade de se envolverem em comportamentos de risco para a saúde. Esta relação pode ser compreendida pela vulnerabilidade aumentada que existe durante a infância e a adolescência, períodos de grande mudança e em que a vivência de experiências adversas pode comprometer o equilíbrio necessário para o desenvolvimento saudável ${ }^{1}$.

Neste sentido, tem-se procurado perceber quais os tipos de experiências que podem constituir adversidade, qual a trajectória desenvolvimental dos indivíduos sujeitos a experiências adversas durante a sua infância, e qual a sua probabilidade de desenvolver patologia física e psicológica na idade adulta.

Por adversidade entende-se a exposição a um conjunto de circunstâncias pouco favoráveis, capazes de interferir com o desenvolvimento normal dos indivíduos ${ }^{2}$. As experiências adversas na infância têm sido definidas não só como atos de perpetração ou omissão dirigidos à criança, mas também como um conjunto de outras condições que afetam o seu ambiente familiar e social ${ }^{1}$.

O Adverse Childhood Experiences Study $(\mathrm{ACE})^{3}$ organizou estas experiências em dez categorias relacionadas com a criança e com o seu ambiente familiar disfuncional, sendo cinco diretamente relacionadas com a criança e cinco relacionadas com o ambiente familiar. Nas experiências contra a criança inserem-se o abuso físico, o abuso emocional e o abuso sexual, mais duas categorias que dizem respeito a atos omitidos à criança e, portanto, a situações de negligência, quer física, quer emocional. Em relação ao ambiente familiar são incluídos o abuso de substâncias, separação ou divórcio parental, doença mental ou suicídio, violência doméstica e prisão.

Apesar da investigação se ter centrado, durante algum tempo, nos efeitos de cada uma destas formas de adversidade em separado, recentemente tem-se reconhecido que estas experiências, para além de frequentes, também tendem a ocorrer simultaneamente e, portanto, a probabilidade de as crianças serem vítimas de mais de um tipo de maus-tratos é elevada ${ }^{3}$. Para além desta coocorrência, sabe-se atualmente que quan- to maior for o número de experiências adversas, maior é o seu efeito no desenvolvimento do indivíduo, tanto ao nível do ajustamento psicológico como ao nível da saúde física ${ }^{4}$.

De facto, as experiências adversas ao longo da vida têm potencial para afetar de forma considerável o funcionamento normal do indivíduo, tanto a nível emocional, como cognitivo, comportamental e fisiológico, dando origem ou exacerbando uma série de sintomas e perturbações mentais. De acordo com Felitti et al. ${ }^{3}$, as perturbações mais comummente associadas às experiências de adversidade são as perturbações de ansiedade, do humor, de personalidade e de abuso de substâncias.

Os acontecimentos de vida traumáticos e adversos têm também um forte impacto ao nível da saúde física, o qual pode ser verificado nos comportamentos de saúde, queixas físicas, utilização dos serviços de saúde, morbilidade e mortalidade ${ }^{5}$.

O grupo de investigação ACE tem realizado vários estudos na tentativa de perceber de que forma é que as experiências adversas ao longo da vida, principalmente durante a infância, se relacionam com a morbilidade e a mortalidade. Neste sentido, os estudos realizados por Felitti et al. ${ }^{3}$ mostraram uma forte relação entre a exposição a essas experiências e o desenvolvimento de problemas físicos. Em termos de morbilidade, os autores verificaram que os indivíduos que relataram quatro ou mais tipos de adversidade durante a infância tinham mais problemas respiratórios, cancro, problemas cardíacos e acidentes vasculares cerebrais, problemas gastrointestinais, diabetes, obesidade, SIDA e fracturas esqueléticas. Do mesmo modo, verificaram que sujeitos com histórias de vida adversas tinham maiores índices de mortalidade.

No que diz respeito aos comportamentos de risco para a saúde, os comportamentos sexuais de risco, como início de atividade sexual precoce, múltiplos parceiros sexuais, maior probabilidade de contrair VIH ou outras doenças sexualmente transmissíveis, maior número de gravidezes na adolescência, e risco superior de violência nas relações íntimas, são apontados como os mais relacionados com a exposição à adversida$\mathrm{de}^{6,7}$. Ou seja, a exposição a várias formas de adversidade durante a infância tem consequências nos comportamentos sexuais dos indivíduos e, quanto maior o número de categorias de experiências adversas vivenciadas, maior a prevalência de doenças sexualmente transmissíveis ${ }^{6}$.

Viver a infância em ambientes familiares onde o consumo de substâncias psicoativas está pre- 
sente num ou em ambos os pais, aumenta a probabilidade de experimentar outras formas de adversidade, assim como de se envolver em comportamentos de consumo mais cedo, e desses comportamentos perdurarem até à idade adulta. Por outro lado, o facto dos indivíduos expostos a situações adversas poderem desenvolver perturbações psiquiátricas, como a Perturbação de Stress Pós Traumático (PTSD) ou a Depressão, deixa-os mais vulneráveis para o consumo de substâncias ${ }^{8}$. Assim, a incidência do consumo de álcool, tabaco e drogas, por parte dos indivíduos que viveram infâncias traumáticas, é mais elevada do que na população em geral ${ }^{9-11}$.

Estudando a exposição à adversidade na infância e as suas consequências ao nível da morbilidade física e psicológica, verificamos que existem populações particularmente expostas a contextos de adversidade, como é o caso da população reclusa ${ }^{12,13}$. Na literatura a população reclusa aparece caracterizada pela elevada prevalência de adversidade ao longo da vida e por um maior envolvimento em comportamentos de risco para a saúde e por estados de saúde, quer física, quer mental, mais degradados.

No que diz respeito à população reclusa feminina, tem-se verificado que estas mulheres apresentam uma elevada taxa de exposição aos mais variados traumas, especialmente ao abuso físico e sexual durante a infância ${ }^{13-15}$, sendo que este pode ter um papel central no desenvolvimento de trajectórias delinquentes. Num estudo efetuado por Mullings et al. ${ }^{16}$ foi verificado que as mulheres que foram abusadas durante a infância são detidas em idades precoces, têm mais probabilidade de viver em condições de marginalidade, apresentam mais consumos de substâncias ilícitas, têm mais histórias de prostituição e envolvem-se num maior número de comportamentos de risco para a saúde do que as mulheres que não tiveram histórias de abuso.

Também na população reclusa masculina, comparativamente à população geral, o consumo de álcool, tabaco e drogas tende a ser mais elevado, sendo que o número de detenções por crimes relacionados com o uso de drogas ilícitas tem vindo a aumentar significativamente ${ }^{17}$. Alguns autores sugerem que o consumo de substâncias psicoativas, assim como outros comportamentos de risco, nomeadamente comportamentos automutilatórios, tentativas de suicídio, entre outras estratégias desadequadas, são formas para regular o afecto e lidar com toda a adversidade vivida por estes indivíduos ${ }^{10,18}$. Ou seja, a exposição à adversidade pode levar a um início precoce do consumo de substâncias e, por sua vez, os indivíduos que consomem mais cedo têm mais probabilidade de continuar a abusar dessas substâncias ao longo da vida, de se envolverem com pessoas delinquentes, participarem em atividades e comportamentos desviantes e, consequentemente, de se envolverem em atos criminais ${ }^{19}$.

Considerando a literatura aqui revista, e dada a relevância da prevalência de fenómenos como adversidade, psicopatologia e comportamentos de risco para a saúde na população reclusa, este estudo tem como objetivos a caracterização das mulheres e dos homens reclusos, no que diz respeito a tais variáveis, e a comparação entre ambos.

\section{Método}

Este estudo foi realizado com 42 indivíduos do sexo feminino e 65 indivíduos do sexo masculino, institucionalizados em estabelecimentos prisionais do norte de Portugal (Tabela 1).

O Questionário Sociodemográfico e História de Vida (de Felitti e Anda ${ }^{20}$; versão portuguesa de Silva e $\mathrm{Maia}^{21}$ ) é composto por duas partes. A primeira é centrada em dados sociodemográficos, enquanto a segunda tem itens organizados em grupos de questões dicotómicas, de escolha múltipla ou resposta breve sobre o estilo de vida e comportamentos de saúde. Este instrumento permitiu averiguar a presença de seis comportamentos de risco para a saúde como: inatividade física, consumo de tabaco, álcool (foi considerado o consumo de álcool enquanto comportamento de risco para a saúde sempre que o sujeito afirmou que bebia mais do que três bebidas alcoólicas por dia), drogas ilícitas, relações sexuais desprotegidas e tentativas de suicídio. O somatório dos comportamentos de risco acima apresentados permitiu calcular um índice de comportamentos de risco para a saúde que pode variar entre 0 e 6 .

O Questionário de História de Adversidade na Infância ${ }^{20,21}$ é na forma de autorrelato para adultos, que pretende avaliar a ocorrência de experiências adversas na infância. Os itens estão organizados de acordo com dez categorias de adversidade: abuso emocional, abuso físico, abuso sexual, negligência física e negligência emocional, exposição à violência doméstica, abuso de substâncias no ambiente familiar, divórcio ou separação parental, prisão de um membro da família, doença mental ou suicídio. A partir do somatório destas dez categorias é possível calcular um índice de adversidade total. É atribuído o 
Tabela 1. Caracterização sociodemográfica dos participantes.

\begin{tabular}{|c|c|c|c|}
\hline Idade & $\begin{array}{c}\text { Mulheres }(\mathrm{N}=42) \\
\text { Média }(\mathrm{DP}) \\
31,8(8,26) \\
\mathbf{N}(\%)\end{array}$ & $\begin{array}{c}\text { Homens }(\mathbf{N}=\mathbf{6 5}) \\
\text { Média }(\mathbf{D P}) \\
35,8(11,71) \\
\mathbf{N}(\%)\end{array}$ & $\begin{array}{l}\mathbf{t}(\mathbf{1 0 5}) \\
1,915 \\
\boldsymbol{X}_{(5)}^{\mathbf{2}}\end{array}$ \\
\hline \multicolumn{4}{|l|}{ Estado Civil } \\
\hline Casado & $15(36)$ & $5(8 \%)$ & $28.61^{* * *}$ \\
\hline União de facto & $11(26)$ & $5(8 \%)$ & \\
\hline Viúvos & 0 & $2(3 \%)$ & \\
\hline Separado & $1(2)$ & 0 & \\
\hline Divorciados & $7(17)$ & $17(26 \%)$ & \\
\hline Solteiros & $8(19)$ & $36(55 \%)$ & \\
\hline \multicolumn{4}{|l|}{ Etnia } \\
\hline Caucasiana & $26(62)$ & $57(88 \%)$ & $14.17^{*}$ \\
\hline Afro-Americana & $1(2)$ & $3(4 \%)$ & \\
\hline Hispânica & $3(7)$ & 0 & \\
\hline Cigana & $12(29)$ & $5(8 \%)$ & \\
\hline \multicolumn{4}{|l|}{ Escolaridade } \\
\hline Menos que 4 anos & $8(19)$ & $5(8 \%)$ & 5.06 n.s. \\
\hline $4^{\circ}$ ano & $10(24)$ & $21(32 \%)$ & \\
\hline $6^{\circ}$ ano & $15(36)$ & $27(41 \%)$ & \\
\hline $9^{\circ}$ ano & $3(7)$ & $7(11 \%)$ & \\
\hline $12^{\circ}$ ano & $4(10)$ & $3(5 \%)$ & \\
\hline For. Univer. & $2(5)$ & $2(3 \%)$ & \\
\hline \multicolumn{4}{|l|}{ Estatuto Profissional } \\
\hline Empregado & $22(5)$ & $52(80 \%)$ & $18.77^{* * *}$ \\
\hline Emp. part-time & 3() & $4(6 \%)$ & \\
\hline Desempregado & $17(4)$ & $5(8 \%)$ & \\
\hline Reformado & 0 & $4(6 \%)$ & \\
\hline
\end{tabular}

${ }^{* *} p=.000{ }^{*} p<.05$

valor "0" caso a adversidade não tenha sido relatada e o valor " 1 " quando a adversidade foi relatada. Note-se que as categorias de abuso físico e emocional e todas as de negligência, que são avaliadas em itens com cinco alternativas de respostas (de nunca ocorreu até muitíssimas vezes), só são consideradas como ocorrentes (valor 1) caso tenham sido experienciadas "muitas vezes" ou "muitíssimas vezes". O valor do índice de adversidade total pode oscilar entre 0 e 10.

Estes dois questionários demonstraram boas características psicométricas, nomeadamente boa consistência temporal, quer nos originais ${ }^{22}$, quer nas versões portuguesas ${ }^{23}$.

O Brief Symptoms Inventory (de Derogatis ${ }^{24}$; adaptado por Canavarro ${ }^{25}$ ) é um instrumento de autorresposta constituído por 53 itens, onde o sujeito deverá classificar o grau em que cada problema o afectou durante a última semana, numa escala tipo likert, cotado desde "nunca" (0) a "muitíssimas vezes" (4). Este instrumento avalia sintomas psicopatológicos em nove dimen- sões básicas (Somatização, Obsessões-Compulsões, Sensibilidade Interpessoal, Depressão, Ansiedade, Hostilidade, Ansiedade Fóbica, Ideação Paranoide e Psicoticismo). O BSI permite ainda calcular um Índice de Sintomas Positivos (ISP), com um ponto de corte de 1.7, acima do qual se consideram valores clínicos de psicopatologia. Os estudos psicométricos da versão portuguesa revelaram que este instrumento apresenta níveis adequados de consistência interna para as nove escalas. Estudos de fiabilidade e validade indicam que este instrumento avalia adequadamente a psicopatologia ${ }^{25}$.

No presente estudo foram retirados os itens que compõem a dimensão de Ansiedade Fóbica, devido ao facto de não se adequarem à condição de reclusão (por exemplo, medo de viajar de autocarro, comboio ou metro).

Após submissão do projeto de investigação ao comité de ética da Direcção Geral dos Serviços Prisionais Portugueses, foi-nos concedida a autorização para a execução do trabalho e agenda- 
da a calendarização da recolha dos dados de forma a não prejudicar a rotina diária do estabelecimento e a manter todo o rigor ético que um trabalho desta dimensão exige.

Cada participante foi chamado aleatoriamente a um gabinete no estabelecimento prisional, sendo-lhe explicado o objetivo do estudo. Caso se mostrasse disponível para participar, preenchia o consentimento informado, como garantia de confidencialidade e anonimato dos seus dados pessoais, para assim poder responder aos questionários individualmente e com a ajuda da investigadora.

A investigadora não tinha qualquer tipo de relação anterior com a população alvo, uma vez que não fazia parte dos quadros clínicos do estabelecimento prisional, estando no mesmo apenas para fins de recolha de dados.

O método de amostragem utilizado foi o método por conveniência, uma vez que foram avaliados todos os sujeitos que aceitaram participar durante o tempo de recolha de dados.

Das 50 mulheres avaliadas, oito não terminaram de responder aos questionários, pelo que foram excluídas. Na população masculina os 65 homens abordados aceitaram participar no estudo, e todos responderam à totalidade das questões.

\section{Resultados}

Os resultados apresentados na Tabela 2 dizem respeito à comparação entre sexos quanto à his- tória de diferentes experiências adversas durante a infância.

Considerando o Índice de Adversidade Total, verificou-se que a média de experiências adversas na população feminina é de $5.05(D P=2.63)$ e, na população masculina, é de $2.69(D P=2.18)$. A realização do teste de Mann -Whitney permitiu verificar diferenças estatisticamente significativas entre os dois $\operatorname{sexos}(Z=-4.33 ; p=.000)$.

As médias do índice de comportamentos de risco para a saúde são $3.07(D P=1.09)$ nas mulheres e $3.02(D P=1.47)$ nos homens. O teste de Mann -Whitney permitiu concluir que não se verificam diferenças estatisticamente significativas $(Z=-.35 ; p=.73)$ entre homens e mulheres relativamente à média de comportamentos de rico para a saúde. A comparação entre homens e mulheres reclusas, no que diz respeito aos diferentes comportamentos de risco para a saúde, está patente na Tabela 3 .

Relativamente à sintomatologia psicopatológica, verificamos que nas mulheres o valor de ISP médio é de $2.7(D P=.45)$, estando todas as participantes acima do ponto de corte, e nos homens o valor de ISP médio é de $2.1(D P=.71)$, estando $75.4 \%$ destes indivíduos acima do ponto de corte para valores clínicos. Em relação às diferentes dimensões da BSI, as participantes femininas apresentaram médias superiores às da população masculina em todas as dimensões, e as diferenças entre estas médias apenas não se revelaram significativas nas dimensões de somatização e hostilidade (Tabela 4).

Tabela 2. Comparação entre mulheres e homens quanto a Experiências Adversas na Infância.

\begin{tabular}{|c|c|c|c|c|c|}
\hline \multirow[b]{2}{*}{ Variável } & \multicolumn{2}{|c|}{ Mulheres $(N=42)$} & \multicolumn{2}{|c|}{ Homens $(N=65)$} & \multirow[b]{2}{*}{$\mathrm{X}_{(1)}^{2}$} \\
\hline & $\mathbf{N}$ & $\%$ & $\mathrm{~N}$ & $\%$ & \\
\hline \multicolumn{6}{|l|}{ Experiências do indivíduo } \\
\hline Abuso Físico & 32 & 76.2 & 18 & 27.7 & $24.11^{* * *}$ \\
\hline Abuso Emocional & 21 & 50.0 & 18 & 27.7 & $5.48^{\prime}$ \\
\hline Abuso Sexual & 11 & 26.2 & 22 & 33.9 & .70 n.s. \\
\hline Negligência Física & 38 & 66.7 & 15 & 23.0 & $20.17^{* * *}$ \\
\hline Negligência Emocional & 19 & 45.2 & 19 & 29.2 & 2.86 n.s. \\
\hline \multicolumn{6}{|l|}{ Disfunção Familiar } \\
\hline Familiar Consumidor de Substâncias & 26 & 61.9 & 27 & 41.5 & $4.23^{\circ}$ \\
\hline Familiar Preso & 21 & 50.0 & 13 & 20.0 & $10.59^{* *}$ \\
\hline Familiar com Doença Mental ou Suicídio na família & 23 & 54.7 & 11 & 16.9 & $16.85^{* * *}$ \\
\hline Divórcio/Separação Parental & 12 & 28.6 & 12 & 18.5 & 1.50 n.s. \\
\hline Mãe Vítima de Violência Doméstica & 19 & 45.2 & 20 & 26.3 & 2.31 n.s. \\
\hline
\end{tabular}


Tabela 3. Comparação entre mulheres e homens em relação a cada um dos Comportamentos de Risco para a Saúde.

\begin{tabular}{|c|c|c|c|c|c|}
\hline \multirow{2}{*}{$\begin{array}{c}\text { Comportamentos } \\
\text { de Risco }\end{array}$} & \multicolumn{2}{|c|}{ Mulheres $(N=42)$} & \multicolumn{2}{|c|}{ Homens $(N=65)$} & \multirow[b]{2}{*}{$\mathrm{X}_{(1)}^{2}$} \\
\hline & $\mathrm{N}$ & $\%$ & $\mathbf{N}$ & $\%$ & \\
\hline Inactividade física & 31 & 73.8 & 37 & 56.9 & 3.14 n.s. \\
\hline Consumo de tabaco & 29 & 69.0 & 51 & 78.5 & 1.19 n.s. \\
\hline Consumo de álcool & 9 & 21.4 & 25 & 38.5 & 3.41 n.s. \\
\hline Consumo de drogas ilícitas & 21 & 50.0 & 39 & 60.0 & 1.04 n.s. \\
\hline Relações sexuais desprotegidas & 24 & 57.1 & 23 & 36.5 & $4.90^{*}$ \\
\hline Tentativa de suicídio & 15 & 35.4 & 21 & 32.3 & .13 n.s. \\
\hline
\end{tabular}

${ }^{*} p<.05$

Tabela 4. Comparação entre mulheres e homens relativamente a cada uma das dimensões psicopatológicas (BSI).

\begin{tabular}{lccr}
\hline \multicolumn{1}{c}{ Dimensão } & $\begin{array}{c}\text { Mulheres }(\mathbf{N}=\mathbf{4 2}) \\
\text { Média (DP) }\end{array}$ & $\begin{array}{c}\text { Homens (N= 65) } \\
\text { Média (DP) }\end{array}$ & Z \\
\hline Somatização & $1.18(.92)$ & $0.47(0.59)$ & $-1,37$ n.s. \\
Obsessões-Compulsões & $1.33(.81)$ & $0.67(0.62)$ & $-4,14^{* *}$ \\
Sensibilidade Interpessoal & $1.18(.93)$ & $0.68(0.74)$ & $-1,97^{*}$ \\
Depressão & $1.73(.99)$ & $1.01(0.73)$ & $-4,90^{* * *}$ \\
Ansiedade & $1.76(.91)$ & $0.87(0.76)$ & $-3,84^{* * *}$ \\
Hostilidade & $1.26(1.10)$ & $0.63(0.67)$ & $-1,86$ n.s. \\
Ideação Paranoide & $1.80(.90)$ & $1.11(0.93)$ & $-3,54^{* * *}$ \\
Psicoticismo & $1.30(.85)$ & $0.78(0.73)$ & $-3,18^{* *}$ \\
\hline
\end{tabular}

${ }^{* * * *} p=.000 ;{ }^{* * *} p<.000 ; \mathrm{p}<<.05$

\section{Discussão}

Os objetivos deste estudo eram conhecer, em reclusos, a prevalência de experiências adversas durante a infância, comportamentos de risco para a saúde e psicopatologia na idade adulta, e perceber as diferenças existentes entre homens e mulheres, referentes a estes três fenómenos.

Quando se considera o total de adversidade, verifica-se que em ambos os sexos a adversidade é bastante prevalente, uma vez que apenas uma mulher e nove homens não relataram qualquer tipo de experiência adversa na infância. Estes valores são claramente superiores aos obtidos num estudo português efectuado por Silva e Maia ${ }^{21}$, com um grupo de sujeitos da população geral, composto por 63 mulheres e seis homens, com uma média de idades de 36.88 anos (com desvio padrão de 10.215 ), uma vez que $31 \%$ da população geral não relatou qualquer tipo de adversidade.

Analisando a diferença entre homens e mulheres reclusos, as mulheres relataram um valor médio de adversidade total significativamente superi- or ao dos homens. Estudos revistos por Suter et al. ${ }^{15}$ vão ao encontro destes resultados, evidenciando que as mulheres reclusas têm histórias de traumas durante a infância mais extensas do que os homens reclusos. Apesar da relevância destes dados, não podemos descurar os valores elevados de desvio padrão, especialmente nos participantes de sexo masculino, sugerindo uma grande variedade de número de experiências de vida adversas.

Os resultados sugerem também que, relativamente às diferentes experiências de adversidade durante a infância, as mulheres relatam mais abuso físico e abuso emocional, bem como negligência e disfunção familiar do que os homens, mas, no que diz respeito ao abuso sexual, e contrariando o que tem vindo a ser descrito na literatura, tal não se verificou. Estudos efetuados com homens e mulheres dependentes de substâncias em situação de reclusão apontam para que as mulheres relatem maior número de casos de abuso físico, emocional e sexual durante a infância do que os homens ${ }^{13}$. Outras investigações referem igualmente que as mulheres que come- 
tem crimes têm passados com história de abusos, essencialmente abuso físico e sexual ${ }^{14,16,26}$. No presente estudo estas diferenças notam-se principalmente a nível do abuso físico, em que as diferenças são altamente significativas, já que o abuso sexual foi mais relatado, ainda que de forma estatisticamente não significativa, pela população masculina. De acordo com Johnson et al. ${ }^{27}$, o abuso sexual em homens detidos por crimes sexuais varia entre os 41 e $43 \%$, e entre os 22 e $23 \%$ nos detidos por outros crimes. Um dos factores que poderá ter contribuído para esta maior prevalência de abuso sexual junto dos homens no nosso estudo é o tipo de crime cometido pelos sujeitos. Ainda que o tipo de crime não tenha sido considerado como variável do estudo, através de estatísticas publicadas pela Direcção Geral dos Serviços Prisionais, sabe-se que no estabelecimento prisional onde foi feita a recolha de dados dos participantes do sexo masculino há uma percentagem elevada de abusadores sexuais.

A elevada incidência de abuso emocional a coexistir com outras formas de abuso vai ao encontro do que é descrito na literatura. A investigação mostra que é frequente a coocorrência de abuso físico e abuso emocional, ou seja, quando as crianças são maltratadas fisicamente tendem a ser maltratadas emocionalmente ${ }^{28}$.

Como tem sido referido, as experiências adversas na infância são um conjunto complexo de experiências altamente inter-relacionadas, raramente ocorrendo de forma isolada ${ }^{3}$, o que significa que a prevalência das experiências adversas no seio familiar pode explicar a elevada comorbilidade com o abuso dirigido ao indivíduo. Neste sentido, torna-se importante realçar o facto de se considerar as experiências adversas na sua totalidade, assim como o seu carácter cumulativo e a influência negativa a curto e longo prazo nos comportamentos, bem-estar emocional e social, e saúde física e mental dos indivíduos ${ }^{3,4,6,9,18}$. No que se refere a comportamentos de risco para a saúde, embora as mulheres reclusas apresentem um Índice de Comportamentos de Risco médio ligeiramente superior ao dos homens, esta diferença não é significativa. $\mathrm{O}$ comportamento em que há diferenças, por as mulheres os relatarem mais, refere-se a relações sexuais desprotegidas. Os dados do nosso estudo estão de acordo com o estudo de Freudenberg et al. ${ }^{12}$, em que verificaram que os homens correm menos risco de prejudicar a sua saúde devido a comportamentos sexuais de risco do que as mulheres. Quanto ao risco de suicídio, cerca de um em cada três parti- cipantes deste estudo referiu pelo menos uma tentativa, o que é um valor bastante elevado. Gunter et al. ${ }^{29}$ referem que as mulheres reclusas apresentam taxas mais elevadas deste comportamento de risco para a saúde do que os homens, o que não se verifica no nosso estudo.

Relativamente à sintomatologia psicopatológica, esta é muitíssimo elevada nas mulheres, mas quando consideramos o número de sujeitos com valores clínicos, também o é nos homens. Notese que apenas nas dimensões de somatização e hostilidade não há diferenças estatisticamente significativas entre os dois sexos. Estes dados confirmam os resultados de Gunter et al. ${ }^{29}$ que constatou uma elevada prevalência de doenças mentais em reclusos face à população em geral e, quando comparados homens com mulheres, estas padecem de mais perturbações psiquiátricas. Também uma revisão efetuada por Suter et al. ${ }^{15}$ demonstrou que inúmeros estudos reúnem consenso relativamente ao facto das perturbações mentais nos estabelecimentos prisionais serem mais comuns nas mulheres do que nos homens.

Tal como pudemos verificar na literatura revista, também neste estudo problemáticas como a adversidade durante a infância, comportamentos de risco para a saúde e perturbação mental apresentam valores elevados junto da população reclusa.

As diferenças entre sexos são mais notórias no que diz respeito aos relatos de adversidade na infância e à sintomatologia psicológica, porém também existem diferenças no que diz respeito aos comportamentos de risco para a saúde, essencialmente ao nível dos comportamentos sexuais de risco. É de salientar que todos os fenómenos em que se destacam diferenças significativas entre sexos são mais prevalentes junto da população reclusa feminina, demonstrando assim uma maior vulnerabilidade desta em termos de saúde física e mental.

Aliando este facto ao conhecimento de que a população feminina tem vindo a aumentar substancialmente nos estabelecimentos prisionais ${ }^{14,26,29}$, emerge a necessidade da investigação se debruçar mais sobre esta e de se pensarem programas específicos de intervenção junto das mulheres reclusas.

Não poderíamos dar por terminadas estas considerações sem antes ressalvar as limitações presentes nesta investigação, nomeadamente o número diminuto de sujeitos nela incluídos e o facto de nos basearmos em instrumentos de autorrelato. 


\section{Colaboradores}

Joana Alves trabalhou na concepção e planeamento do estudo, na pesquisa bibliográfica, na recolha, análise e interpretação dos dados, assim como na redacção do artigo; Ana Dutra trabalhou na pesquisa bibliográfica e na recolha de dados; Ângela Maia trabalhou na concepção e planeamento do estudo, na pesquisa bibliográfica, na análise e interpretação dos dados, assim como na redacção do artigo e na revisão crítica do mesmo.

\section{Referências}

1. Clemmons J, DiLillo D, Martinez I, DeGue S, Jeffcott M. Co-occurring forms of child maltreatment and adult adjustment reported by Latina college students. Child, Abuse Negl 2003; 27(7):751-767.

2. Rizzini I, Dawes A. Editorial on cultural diversity and childhood diversity. Childhood 2001; 8:315-321.

3. Felitti V, Anda R, Nordenberg D, Williamson D, Spitz A, Edwards V, Koss MP, Marks JS. Relationship of childhood abuse and household dysfunction to many of the leading causes of death in adults: The Adverse Childhood Experiences (ACE) Study. Am J Prev Med 1998; 14(4):245-258.

4. Dong M, Anda RF, Dube SR, Giles WH, Felitti JV. The relationship of exposure to childhood sexual abuse to other forms of abuse, neglect, and household dysfunction during childhood. Child Abuse Negl 2003; 27(6):625-639.

5. Maia A. Trauma e Saúde. In: Ribeiro J, Leal I, organizadores. Actas do $5^{\circ}$ Congresso Nacional de Psicologia da Saúde. Lisboa: Fundação Calouste Gulbenkian; 2004. p. 35-41.

6. Hillis SD, Anda RF, Felitti VJ, Nordenberg D, Marchbanks PA. Adverse Childhood Experiences and Sexual Transmitted Diseases in Men and Women: A Retrospective Study. Pedriatrics 2000; 106(1):E11.

7. Anda RF. Whitfield CL, Felitti JV, Chapman D, Edwards VJ, Dube SR, Williamson D. Adverse childhood experiences, alcoholic parents, and later risk of alcoholism and depression. Psychiatr Serv 2002; 53(8):1001-1009.

8. Connor K, Butterfield M. Posttraumatic Stress Disorder. Focus [Internet]. 2003; 1(3):247-262. [acessado 2012 fev 20]. Disponível em: http://focus. psychiatryonline.org/cgi/reprint/1/3/247.

9. Dube S, Anda R, Felitti V, Edwards V, Croft J. Adverse childhood experiences and personal alcohol abuse as an adult. Addict Behav 2002; 27(5):713-725.

10. Anda RF, Croft JB, Felitti VJ, Nordenberg D, Giles WH, Williamson DF, Giovino GA. Adverse childhood experiences and smoking during adolescence and adulthood. JAMA 1999; 282(17):1652-1658.

11. Dube SR, Felitti VJ, Dong M, Chapman DP, Giles WH, Anda RF. Childhood abuse, neglect, and household dysfunction and the risk of illicit drug use: The adverse childhood experiences. Pediatrics 2003; 111(3):564-572.

12. Freuddenberg N, Moseley J, Labriola M, Daniels J, Murrill C. Comparison of Health and Social Characteristics of People Leaving New York City Lails by Age, Gender, and Race/Ethnicity: Implications for Public Health Interventions. Public Health Rep 2007; 122(6):733-743. 
13. Messina N, Grella C. Childhood Trauma and Women's Health Outcomes in a California Prison Population. Am J Public Health 2006; 96(10):1842-1848.

14. Green BL, Miranda J, Daroowalla A, Siddique J. Trauma exposure, mental health functioning, and program needs of women in jail. Crime and Delinquency 2005; 51(1):133-151.

15. Suter J, Byrne M, Byrne S, Howells K, Day A. Anger in Prison: women are different from men. Personality and Individual Differences 2002; 32(6):1087-1100.

16. Mullings JL, Marquart JW, Brener VE. Assessing the relationship between child sexual abuse and marginal living conditions on HIV/AIDS - related risk behaviour among women prisoners. Child Abuse Negl 2000; 24(5):677-688.

17. Diamond PM, Wang EW, Holzer CE, Thomas C, Cruser A. The prevalence of mental illness in prison. Adm Policy Ment Health 2001; 29(1):21-40.

18. Dube S, Anda R, Felitti V, Chapman D, Williamson D, Giles W. Childhood abuse, household dysfunction, and the risk of attempted suicide throughout the life span. Findings from the adverse childhood experiences study. JAMA 2001; 286(24):3089-3096.

19. Johnson D. Age of illicit drug initiation. Trends and Issues in Crime and Criminal Justice Series. [Internet]. 2001;201:1-6. [acessado 2012 fev 20]. Disponível em: http://www.aic.gov.au/publications/tandi/ ti201.pdf

20. Felitti VJ, Anda RF, Nordenberg D, Williamson DF, Spitz AM, Edwards V. Relationship of childhood abuse and household dysfunctions to many of the leading causes of death in adults: The adverse childhood study. American J Preventive Medicine 1998; 14: 245-258.

21. Silva SSP, Maia AC. História de adversidade na família e queixas de saúde - um estudo comparativo entre obesos e não obesos. In: Pereira MG, Simães C, McIntyre MT, Editores. Actas do II Congresso de Família, Saúde e Doença. Braga: Universidade do Minho; 2007.

22. Dube SR, Williamson DF, Thompson T, Felitti VJ, Anda RF. Assessing the reliability of retrospective reports of adverse childhood experiences among adult $\mathrm{HMO}$ members attending a primary care clinic. Child Abuse \& Neglect 2004; 28(7):729-737.

23. Correia L. Consistência do auto-relato de experiências de vida adversas, sintomatologia psicológica e física, $e$ comportamentos de risco para a saúde em jovens sinalizados na infância [tese]. Braga: Universidade do Minho; 2012.
24. Derogatis LR, Lopez MC, Zinzeletta EM. Clinical Applications of the DSFI in the Assessment of Sexual Dysfunctions. In: Brown R, Roberts E, Editors. Advances in the Understanding and Treatment of Sexual Disorders. New York: PNA Publishing; 1988.

25. Canavarro MC. Inventário de Sintomas Psicopatológicos - B.S.I. In: Simões MR, Gonçalves M, Almeida LS, editores. Testes e Provas Psicológicas em Portugal. Braga: APPORT; 1999. p. 95-109.

26. Lewis CF. Post-traumatic stress disorder in HIVpositive incarcerated women. J Am Acad Psychiatry Law 2005; 33(4):455-464.

27. Johnson R, Ross M, Taylor W, Williams M, Carvajal R, Peters R. Prevalence of childhood sexual abuse among incarcerated males in county jail. Child Abuse Negl 2006; 30(1):75-86.

28. Figueiredo B. Maus-Tratos à Criança e ao Adolescente (I): Situação e Enquadramento da Problemática. Psicologia: Teoria Investigação e Prática 1998; 3:5-20.

29. Gunter T, Arndt S, Wenman G, Allen J, Loveless P, Sielene B, Black DW. Frequency of mental and addictive disorders among 320 Men and women entering the Iowa prison system: Use of the MINIPlus. J Am Acad Psychiatry Law 2008; 36(1):27-34.

Artigo apresentado em 01/03/2012

Aprovado em 27/03/2012

Versão final apresentada em 29/03/2012 
